\title{
Caracterización de un músculo artificial de nylon y su implementación en una articulación de un robot manipulador serial
}

\author{
Testing of a nylon artificial muscle and its implementation in a joint of a serial \\ manipulator robot
}

\author{
ZAMUDIO-MARTÍNEZ, Patricio $\dagger^{*} \&$ AGUILAR-SALAZAR, Alejandro Tonatiuh
}

Universidad Nacional Autónoma de México

ID $1^{\text {er }}$ Autor: Patricio, Zamudio-Martínez / ORC ID: 0000-0003-3378-5206, CVU CONACYT ID: 241062

ID $1^{\text {er }}$ Coautor: Alejandro Tonatiuh, Aguilar-Salazar / ORC ID: 0000-0003-3441-7587, CVU CONACYT ID: 840928

DOI: 10.35429/JOES.2019.20.6.1.7

Recibido 9 de Julio, 2019; Aceptado 30 de Septiembre, 2019

\begin{abstract}
Resumen
En este artículo se presenta el estudio y la caracterización de un músculo de nylon, para validar su desempeño, definir sus propiedades, aplicando cargas sobre el mismo y sometiéndolo a estímulos térmicos. Se reportan los resultados de la implementación del músculo de nylon en una articulación de un mecanismo rígido, de un manipulador serial, sin embargo, el difícil control térmico que posee el músculo hace que el choque térmico (la temperatura ambiente y la aplicada) desgaste en cierto punto al músculo, agotando sus propiedades, llegando al punto de quemar el material o recibiendo una respuesta muy lenta por parte de este. Se comprueba que el material sólo puede soportar cargas en suspensión mientras que su implementación en un mecanismo rígido aún es cuestionable para su aplicación en sistemas rígidos. Se observa que una característica importante de los músculos de nylon es su increíble capacidad de contraer cargas que son superiores al mismo peso del músculo y que por medio de un estímulo térmico pueden contraerse gracias a su propiedad anisotrópica, así como el material es sumamente atractivo para la aplicación de la robótica blanda y materiales inteligentes.
\end{abstract}

Robótica blanda, Actuadores Blandos, Músculos Artificiales de Nylon

\begin{abstract}
In this paper we present the study and description of a nylon muscle, to validate its performance and define its properties, applying loads on it and subjecting it to thermal stimuli. The results of the implementation of the nylon muscle are reported in a joint of a rigid mechanism, of a serial manipulator, however, the difficult thermal control that the muscle possesses makes the thermal shock (the ambient temperature and the applied one) wear at a certain point to the muscle, exhausting its properties or a very slow response from it. Testing that the material can only be used in the suspension of the load in the application of rigid systems. It is observed that an important feature of nylon muscles is their incredible capacity to contract loads that are higher than the same weight of the muscle and that by means of a thermal stimulus can be contracted thanks to its anisotropic property, just as the material is extremely attractive for the application of soft robotics and intelligent materials
\end{abstract}

Soft Robotics, Soft Actuators, Nylon Artificial Muscle

Citación: ZAMUDIO-MARTÍNEZ, Patricio \& AGUILAR-SALAZAR, Alejandro Tonatiuh. Caracterización de un músculo artificial de nylon y su implementación en una articulación de un robot manipulador serial. Revista de Sistemas Experimentales. 2019. 6-20: 1-7

\footnotetext{
* Correspondencia del Autor (pmz_rofi@comunidad.unam.mx)

$\dagger$ Investigador contribuyendo como primer autor.
} 


\section{Introducción}

La robótica blanda o también conocida como Soft Robotic, es una nueva rama de la robótica en la cual los componentes están esencialmente hechos de materiales blandos y estructuras activas monolíticas (o continuas), esto permite una mejor interacción en su entorno que los robots que están hechos de materiales rígidos. La robótica blanda al estar constituida de materiales blandos causa impacto en las áreas que requieren de materiales sensibles e inteligentes, las aplicaciones médicas, interacción social y la manipulación de objetos frágiles serán de las áreas que la robótica blanda asistirá y tendrá relevancia para realizar tareas que la robótica rígida aún está limitada. (Miriyev, Stack et al. 2017, Campbell 2018, Coyle, Majidi et al. 2018).

A medida que la robótica blanda ha evolucionado, a la par sensores y actuadores compuestos de materiales flexibles e inteligentes surgen de la necesidad de crear sistemas discretos y capaces de realizar movimientos en entornos variables y estos puedan adaptarse al entorno, (Paik 2015, Campbell 2018). Los actuadores blandos están compuestos de polímeros capaces de expandirse y contraerse, efectuando movimientos lineales o rotatorios, (Kim, Yang et al. 2015).

\section{Actuadores blandos actuados eléctricamente}

La esencia de los actuadores blandos actuados eléctricamente es someter un polímero que sirve como dieléctrico entre dos nodos, esto crea un campo magnético entre los dos nodos, haciendo que el dieléctrico por la carga de energía eléctrica sufra una deformación. Actuador blando "elastómeros dieléctricos son materiales aislantes blandos. Se deforman por fuerzas eléctricas cuando se usan en un condensador entre dos electrodos mecánicamente compatibles.

Los elastómeros dieléctricos son un subgrupo de polímeros electro activos, materiales utilizados en transductores electromecánicos" (Bauer 2014). Se han investigado muchas clases de materiales para este tipo de aplicaciones incluyendo acrilatos, siliconas, uretanos cauchos en estado látex ABS y diferentes copolímeros, para su uso como actuadores o músculos artificiales, (Petralia and Wood 2010, Gonzalez-Jimenez 2015).

\section{Actuadores \\ blandos \\ actuados neumáticamente}

Los actuadores blandos actuados neumáticamente son de los sistemas de músculos artificiales en desarrollo entorno a robots blandos, (Yang, Verma et al. 2016). Menciona que un sistema de actuador neumático blando es accionado por el inflado de una red neumática de pequeños canales dentro del material elastómero, que producen contracciones y expansiones en los materiales cuando el sistema es actuado por los flujos de gases comprimidos. Las limitantes de estos tipos de actuadores es que su accionamiento es relativamente lento, por lo que se requieren cantidades grandes de aire comprimido para poder accionar todo el sistema y el flujo del gas comprimido pueda llegar a todos los canales de los materiales elastómeros.

\section{Actuadores blandos actuados hidráulicamente}

Los actuadores actuados hidráulicamente poseen una gran cantidad de energía que se transfiere a través de un sistema que conecta con las partes para ejecutar el movimiento del actuador lo que provoca una alta densidad de potencia, dicho sistemas por lo general están acompañados de maquinaria pesada, debido al gran trabajo y energía que estos brindan al accionarse, (Acome, Mitchell et al. 2018).

\section{Actuadores blandos actuados térmicamente}

Los actuadores accionados térmicamente son los prototipos de músculos artificiales prometedores debido a su simpleza en su funcionamiento y los materiales inteligentes, dentro de los sistemas de actuadores térmicos definimos a un material inteligente que posee memoria de forma al someterse a una diferencia de temperatura, ya que esto le permite al material deformarse $y$ volver a su estado inicial sin dañar su estructura.

\section{Actuadores blandos de Nylon}

En el área de los materiales inteligentes, el nylon presenta propiedades termoplásticas lo que permite que se deforme con un estímulo externo como el calor (Haines 2015) la electricidad por efecto joule (Mirvakili and Hunter 2016) (Mirvakili, Ravandi et al. 2014) o incluso por un sistema que controla el agua a través del músculo, (Wu, Andrade et al. 2015). 
En la actualidad las fibras de nylon y polietileno son grandes precursores de las fibras musculares artificiales, ya que permite combinar la contracción que ocasiona la inducción térmica reversible en dirección de la fibra, la gran expansión térmica volumétrica y la gran anisotropía en los cambios dimensionales inducidos térmicamente proporcionan un golpe muscular. Estas fibras precursoras están compuestas de cadenas poliméricas flexibles que están altamente orientadas en la dirección de la fibra.

La contracción térmica resultante de las fibras de nylon puede llegar a representar un $4 \%$ similar a los cables de memoria de forma de los Ni-Ti, (Yin, Zhou et al. 2018). Para que las fibras de nylon tengan una carrera de tracción, la fibra de nylon se somete a torsión al punto de que se convierta en un espiral, dándole el aspecto de un pequeño resorte, (Haines 2015). La Figura 1 muestra el ejemplo de un músculo de nylon retorcido.

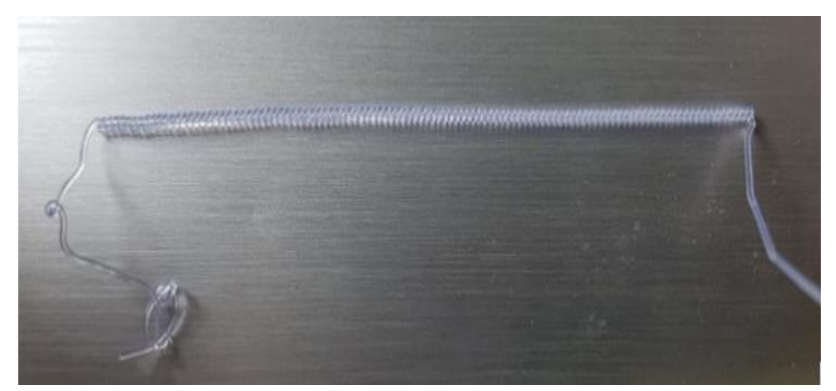

Figura 1 Músculo de nylon retorcido

En la actualidad estos músculos de nylon están accionados a través de 3 sistemas, sistema de agua ( $\mathrm{Wu}$, Andrade et al. 2015), aire caliente (Haines 2015) y el efecto Joule (Mirvakili and Hunter 2016) teniendo gran carrera de recorrido, dichos sistemas consisten en calefacción y enfriamiento, el reto para todos los sistemas que activan el músculo artificial de nylon es someter el material a una temperatura tal que la contracción del músculo se efectué, al tiempo que vuelva a su temperatura inicial cambiando a su estado original del músculo en cuestión de segundos. Los músculos de nylon poseen capacidades de cargas y de resistencia a la deformación semejantes o incluso superiores a los músculos biológicos y que en comparación compiten con otros músculos artificiales utilizados para la elaboración de actuadores blandos (Músculos artificiales eléctricos: elastómeros dieléctricos.
Músculos artificiales neumáticos: "McKibben Artificial Muscles", "VAMP", "Pneu net". músculos artificiales hidráulicos: "Hasel". y etc).

La necesidad de caracterizar y estudiar el desempeño de los músculos artificiales de nylon es brindar en el futuro la posibilidad de controlar mecanismos rígidos (manipuladores seriales, paralelos o híbridos) o blandos (robots continuos) de manera discreta aprovechando espacios en su implementación, sin la necesidad de mantenimiento como los actuadores convencionales y controlar a través de entornos variables, además de tener una mejor respuesta de actuación casi intuitiva.

Por lo que se parte del cuestionamiento si es posible que las características mecánicas de un músculo artificial de nylon puedan efectuar un movimiento lineal parecido a los actuadores lineales convencionales en la implementación de una articulación de un manipulador serial. Los actuadores blandos de nylon se encuentran en etapas de pruebas, debido a que el control y el nivel de respuesta del material aún resulta ser difícil de controlar.

Este articulo presenta el estado del arte de los actuadores blandos, la fabricación del músculo de nylon, la caracterización y estudio de un actuador blando de nylon (músculo artificial) su comportamiento mecánico y su desempeño como un actuador lineal, una aplicación en la implementación de un eslabón en una articulación de un manipulador serial, la realización de pruebas, la implementación del músculo y los resultados que se obtuvieron.

\section{Fabricación del músculo artificial de nylon}

Del artículo "Artificial Muscles From Line And Sewing Thread" (Haines 2015) se tomaron las técnicas para la fabricación del músculo de nylon enroscado, este consiste en someter el filamento de nylon a una tensión tal que se ejerce un torque sobre la misma, modificando su estructura y obteniendo de esta la espiral característica que poseen los músculos de nylon enroscados. Para la construcción del músculo de nylon se, tensó el filamento de nylon a un extremo y se aplicó el torque hasta obtener la espiral característica, posteriormente este músculo retorcido se introdujo en agua caliente para evitar tenciones internas en el proceso de fabricación. 
Otros autores optaron por la construcción de un dispositivo que se dedica a fabricar músculos de nylon, (Semochkin 2016).

\section{Pruebas de alargamiento del músculo}

Ya fabricado el músculo artificial de nylon, se sometió a una prueba de alargamiento, esto permite conocer la magnitud de elongación del material antes de sufrir una deformación por la carga suministrada. En la Figura 2 se aprecia el músculo de Nylon sometido a diferentes cargas.

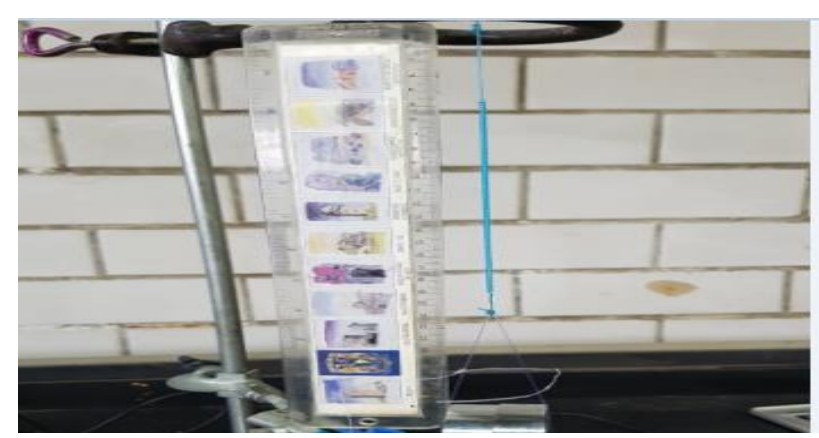

Figura 2 Músculo artificial de nylon sometido a carga

En la tabla 1 se muestra el alargamiento del músculo con respecto al peso medido en newtons.

\begin{tabular}{|r|r|}
\hline Alargamiento (m) & Fuerza (N) \\
\hline 0.085 & 0 \\
\hline 0.087 & 0.667 \\
\hline 0.090 & 1.334 \\
\hline 0.095 & 1.978 \\
\hline 0.097 & 2.002 \\
\hline 0.105 & 2.669 \\
\hline 0.113 & 3.337 \\
\hline 0.120 & 3.957 \\
\hline 0.143 & 5.935 \\
\hline 0.155 & 7.914 \\
\hline 0.164 & 9.893 \\
\hline
\end{tabular}

Tabla 1 Alargamiento y fuerza colocada en el músculo artificial de nylon

Para el estudio de los músculos de nylon se analizan sus propiedades mecánicas para conocer su comportamiento y aprovechar al máximo las características, estos se destacan por sus capacidades de carga altas en proporción a su tamaño e histéresis.

\section{Características mecánicas del músculo}

Se utiliza la teoría de los resortes para definir los parámetros mecánicos del músculo, ya que parte de la configuración de los músculos artificiales de nylon se comportan de la misma manera.
Ley de Hooke. Establece que el alargamiento $\left(x-x_{0}\right)$ del músculo será directamente proporcional al módulo de la fuerza aplicada, donde $(k)$ es la constante del resorte.

$$
F=-k\left(x-x_{0}\right)
$$

Módulo de Young. Mide la resistencia del músculo del nylon $(\sigma)$ al ser deformado elásticamente $(\varepsilon)$.

$E=\frac{\sigma}{\varepsilon}$

Potencia. Es la cantidad de trabajo $(T)$ efectuado sobre una unidad de tiempo $(t)$.

$P=\frac{T}{t}$

Energía cinetica $E_{C}$, es la energia que posee un cuerpo a causa de su movimiento, donde $(m)$ es la masa y $(v)$ la velocidad.

$E_{C}=\frac{1}{2} m v^{2}$

Energía potencia elástica $(U)$. Es la energía ganada al deformar un resorte $(k)$ es una constante y $(x)$ el desplazamiento.

$U=\frac{1}{2} k x^{2}$

Índice de muelles helicoidales $(C)$. Este parámetro permite saber por medio del valor de los diámetros (diámetro del músculo $\mathrm{D}_{\mathrm{E}} \mathrm{y}$ diámetro del filamento $\mathrm{D}_{\mathrm{I}}$ ) del músculo la rigidez del músculo.

$C=\frac{D_{E}}{D_{I}}$

A partir de las anteriores ecuaciones, puede conocer los parámetros mecánicos del músculo de nylon. En la Tabla 2 se muestra las características mecánicas del músculo.

\begin{tabular}{|l|r|}
\hline Ley de Hooke & $197.8 \frac{\mathrm{N}}{\mathrm{m}}$ \\
\hline Módulo de Young & $173.55 \frac{\mathrm{N}}{\mathrm{m}^{2}}$ \\
\hline Potencia & $0.0049465 \mathrm{Watt}$ \\
\hline Energía cinética & $0.009893[\mathrm{~N} * \mathrm{~m}]$ \\
\hline Energía potencial elástica & $0.00989[\mathrm{~N} * \mathrm{~m}]$ \\
\hline Índice de muelles helicoidales. & 3 \\
\hline
\end{tabular}

Tabla 2 Características Mecánicas del Músculo Artificial de Nylon

ZAMUDIO-MARTÍNEZ, Patricio \& AGUILAR-SALAZAR, Alejandro Tonatiuh. Caracterización de un músculo artificial de nylon y su implementación en una articulación de un robot manipulador serial. Revista de Sistemas Experimentales. 2019 


\section{Pruebas de control del músculo}

Se utilizó una pistola de calor, la cual proporciona una temperatura de $300^{\circ} C$, se colocó el músculo en suspensión soportando una carga de $2.669 \mathrm{~N}$. Posteriormente se utilizó la pistola aproximando la boquilla en el músculo. Esto dio como resultado una contracción por parte del músculo artificial de nylon aproximadamente de $0.02 \mathrm{~m}$ levantando la carga de $2.669 \mathrm{~N}$ en un tiempo de 2 a 3 segundos. Ver Figura 3 a) y 3 b).

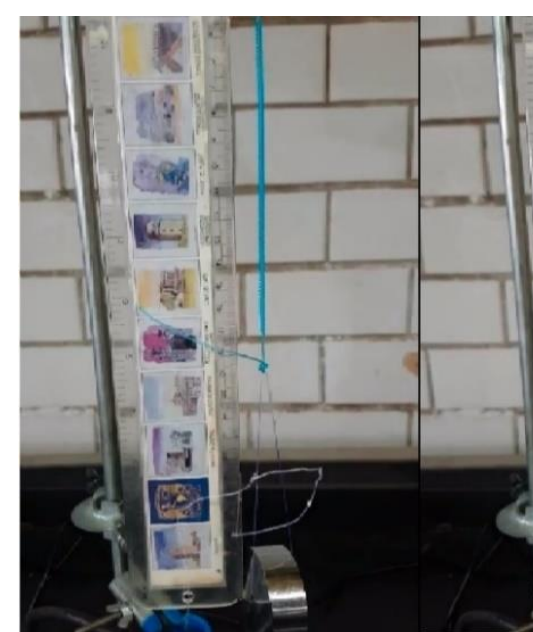

a)

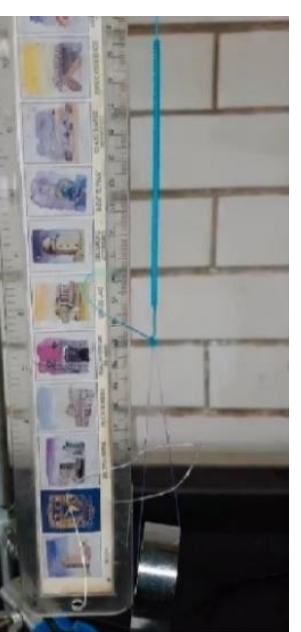

b)
Figura 3 Contracción del músculo de nylon, a) sin calor, b) con calor

Una vez obtenidos los parámetros mecánicos del músculo artificial de nylon, se procedió a evaluar la magnitud de la fuerza necesaria para poder mover la articulación del manipulador serial por medio de un dinamómetro de $1 \mathrm{~kg} * f$ ó $10 \mathrm{~N}$. En esta prueba se obtuvo una fuerza necesaria de aproximadamente $250 g r^{*} f$, para poder desplazar la articulación del brazo del manipulador. Ver Figura 4.

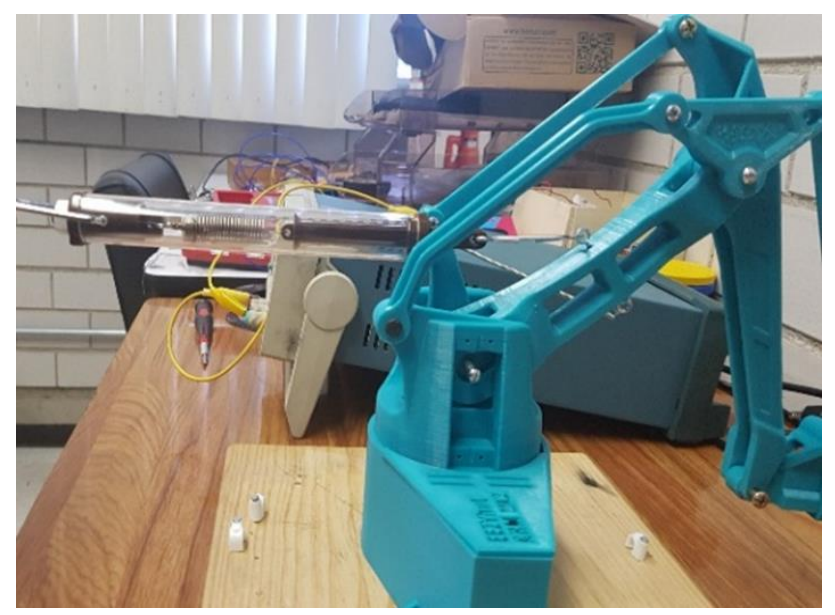

Figura 4 Prueba de fuerza en articulación con dinamómetro

ISSN -2410-3950

ECORFAN $®$ Todos los derechos reservados

\section{Implementación de un manipulador serial}

Durante la implementación del músculo en una articulación del manipulador serial, se observa el movimiento de este al momento de suministrar calor al músculo, obteniendo un desplazamiento angular de aproximadamente $10^{\circ} \mathrm{C}$ por $2 \mathrm{~cm}$ de contracción del músculo de Nylon. Ver figura 5.

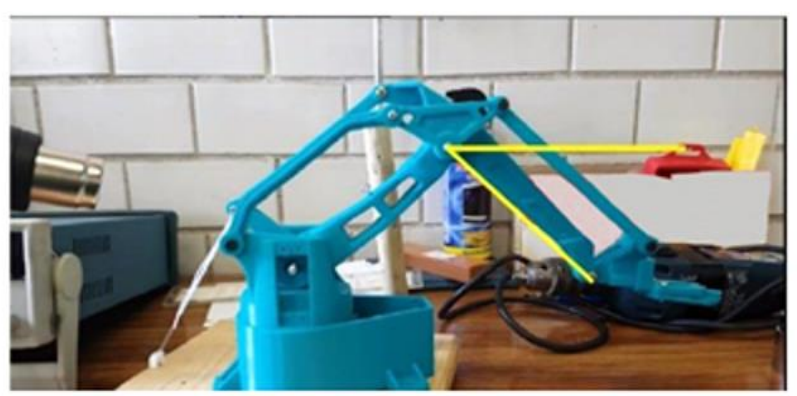

Figura 5 Actuación del músculo dentro de articulación de un manipulador serial

\section{Resultados}

La realización de las pruebas mecánicas del músculo artificial de nylon mostró los parámetros mecánicos de los cuales están constituidos estos músculos, se logró observar y medir el comportamiento del músculo de nylon al estar sometido a cargas y al control de la contracción del músculo por medio del calor suministrado al mismo, teniendo una respuesta entre 2 y 3 segundos, contrayéndose a $2 \mathrm{~cm}$ y soportando cargas aproximadas a los $9.89 \mathrm{~N}$.

Las pruebas realizadas a este tipo de actuadores brindaron la oportunidad de conocer mejor la configuración de este, tomando pruebas para conocer sus capacidades mecánicas y aplicándolos en una articulación de un manipulador son elementos que permiten conocer los alcances que pueden tener estos actuadores para la aplicación en la robótica.

Se obtuvieron resultados interesantes con respecto en la actuación en los manipuladores, sin embargo, el músculo artificial de nylon al ser sometido a diez pruebas, se vio una deformación a causa del contacto directo de la temperatura de la pistola de calor, esto causaba una perdida en la tensión del músculo y por ende la contracción del músculo disminuye. Para trabajo futuro se espera mejorar el control de la temperatura suministrada en un medio controlado para la actuación segura del actuador. 


\section{Agradecimientos}

Agradezco en lo que corresponde a la DGAPA, por el apoyo brindado para la realización de este trabajo, a través del proyecto UNAM-DGAPAPAPIME-PE112319: "Desarrollo de material didáctico y banco de pruebas para el fortalecimiento de la enseñanza en instrumentación y control, aplicando tecnologías IoT y Robótica."

\section{Conclusiones}

Los resultados en la implementación del músculo de nylon en la articulación del robot manipulador serial se observa que el difícil control térmico que posee el músculo hace que el choque térmico y la temperatura ambiente desgasten al músculo agotando sus propiedades. Se comprueba que el material sólo puede soportar cargas en suspensión mientras que su implementación en un mecanismo rígido aún es cuestionable para su aplicación en sistemas rígidos.

El estudio y la aplicación de este tipo de elementos blandos permiten el desarrollo de sistemas discretos y capaces de trabajar bajo diferentes tipos de estímulos dependiendo del entorno. Se observa que una característica importante de los músculos de nylon es su increíble capacidad de contraer cargas que son superiores al mismo peso del músculo. Por lo tanto, el material es sumamente atractivo para la aplicación de la robótica blanda y materiales inteligentes.

\section{Referencias}

Acome, E., S. K. Mitchell, T. G. Morrissey, M. B. Emmett, C. Benjamin, M. King, M. Radakovitz and C. Keplinger (2018). "Hydraulically amplified self-healing electrostatic actuators with muscle-like performance." Science 359(6371): 61-65.

Bauer, S. (2014). Dielectric Elastomers. Encyclopedia of Polymeric Nanomaterials. S. Kobayashi and K. Müllen. Berlin, Heidelberg, Springer Berlin Heidelberg: 1-9.

Campbell, S. (2018). "The Robotics Revolution Will Be Soft: Soft Robotics Proliferate-Along with Their Sources of Inspiration." IEEE Pulse 9(3): 19-24.
Coyle, S., C. Majidi, P. LeDuc and K. J. Hsia (2018). "Bio-inspired soft robotics: Material selection, actuation, and design." Extreme Mechanics Letters 22: 51-59.

Gonzalez-Jimenez, A. R. (2015). Elastómeros dieléctricos para su uso como actuadores o músculos artificiales. Plásticos Modernos. Vol. 109.: 14-17.

Haines, C. S. (2015). Artificial muscles from fishing line and sewing thread, ProQuest LLC.

Kim, J.-H., W. Yang, J. Jo, P. Sincak and H. Myung (2015). Robot Intelligence Technology and Applications 3. Results from the 3rd International Conference on Robot Intelligence Technology and Applications. Cham, sl: Springer International Publishing (Advances in Intelligent Systems and Computing, 345). Online verfügbar unter http://dx. doi. org/10.1007/978-3-319-16841-8. II, Springer.

Miriyev, A., K. Stack and H. Lipson (2017). "Soft material for soft actuators." Nature Communications 8(1): 596.

Mirvakili, S. M. and I. W. Hunter (2016). Bending artificial muscle from nylon filaments. Electroactive Polymer Actuators and Devices (EAPAD) 2016, March 21, 2016 - March 24, 2016, Las Vegas, NV, United states, SPIE.

Mirvakili, S. M., A. R. Ravandi, I. W. Hunter, C. S. Haines, N. Li, J. Foroughi, S. Naficy, G. M. Spinks, R. H. Baughman and J. D. W. Madden (2014). Simple and strong: Twisted silver painted nylon artificial muscle actuated by Joule heating. Electroactive Polymer Actuators and Devices, EAPAD 2014, March 10, 2014 - March 13, 2014, San Diego, CA, United states, SPIE.

Paik, J. (2015). Soft Robotics: Transferring Theory to Application," Soft Components for Soft Robots", Springer.

Petralia, M. T. and R. J. Wood (2010). Fabrication and analysis of dielectric-elastomer minimum-energy structures for highlydeformable soft robotic systems. 2010 IEEE/RSJ International Conference on Intelligent Robots and Systems. 
Semochkin, A. N. (2016). A device for producing artificial muscles from nylon fishing line with a heater wire. 2016 IEEE International Symposium on Assembly and Manufacturing (ISAM).

Wu, L., M. J. d. Andrade, R. S. Rome, C. Haines, M. D. Lima, R. H. Baughman and Y. Tadesse (2015). Nylon-muscle-actuated robotic finger. SPIE Smart Structures and Materials + Nondestructive Evaluation and Health Monitoring, SPIE.

Yin, H., J. Zhou, J. Li and V. S. Joseph (2018). "Fabrication and Properties of Composite Artificial Muscles Based on Nylon and a Shape Memory Alloy." Journal of Materials Engineering and Performance 27(7): 3581-3589. 